\title{
An explicit version of the correspondence between Noetherian modules with Cartier maps and Artinian modules with Frobenius maps
}

\author{
Mehmet Yeşil \\ Department of Mathematics, Faculty of Science and Letters, Batman University, Batman, Turkey
}

\begin{abstract}
In this paper, we introduce an explicit version of the correspondence between Noetherian modules with Cartier maps and Artinian modules with Frobenius maps which recovers the correspondence given by R. Y. Sharp and Y. Yoshino, and the correspondence given by M. Blickle and G. Böckle.
\end{abstract}

Mathematics Subject Classification (2010). 13C60, 13F25, 13A35

Keywords. Cartier maps, Frobenius maps

\section{Introduction}

Throughout this paper our general assumption on $R$ is to be a commutative Noetherian regular ring of prime characteristic $p$. Let $e$ be a positive integer. Let $f: R \rightarrow R$ be the Frobenius homomorphism defined by $f(r)=r^{p}$ for all $r \in R$, whose $e$-th iteration is denoted by $f^{e}$. If the Frobenius homomorphism is finite, $R$ is said to be $F$-finite. Let $M$ be an $R$-module. $F_{*}^{e} M=\left\{F_{*}^{e} m \mid m \in M\right\}$ denotes the Abelian group M with the induced $R$-module structure via the $e$-th iterated Frobenius and it is given by

$$
r F_{*}^{e} m=F_{*}^{e} r^{p^{e}} m \text { for all } m \in M \text { and } r \in R .
$$

In particular, $F_{*}^{e} R$ is the Abelian group $R$ with the induced $R$-module structure

$$
r F_{*}^{e} s=F_{*}^{e} r^{p^{e}} s \text { for all } r, s \in R .
$$

An $e$-th Frobenius map on $M$ is an $R$-linear map $\phi: M \rightarrow F_{*}^{e} M$, equivalently an additive map $\phi: M \rightarrow M$ such that $\phi(r m)=r^{p^{e}} \phi(m)$ for all $r \in R$ and $m \in M$. Let $R\left[X ; f^{e}\right]$ be the skew-polynomial ring whose multiplication is subject to the rule $X r=f^{e}(r) X=r^{p^{e}} X$ for all $r \in R$. Notice that defining an $e$-th Frobenius map on $M$ is equivalent to endowing $M$ with a left $R\left[X ; f^{e}\right]$-module structure extending the rule $X m=\phi(m)$ for all $m \in M$, where $X(r m)=\phi(r m)=r^{p^{e}} \phi(m)=r^{p^{e}} X m=f^{e}(r) X m=(X r) m$ for all $r \in R$ and $m \in M$.

An $e$-th Cartier map on $M$ is an $R$ linear map $C: F_{*}^{e} M \rightarrow M$, equivalently an additive map $C: M \rightarrow M$ such that $r C(m)=C\left(r^{p^{e}} m\right)$ for all $r \in R$ and $m \in M$. An $R$-module $M$ is called a Cartier module if it is equipped with a Cartier map. Notice that defining an $e$-th Cartier map on $M$ is equivalent to endowing $M$ with a right $R\left[X ; f^{e}\right]$-module structure

Email address: mehmet-yesil@outlook.com

Received: 02.05.2019; Accepted: 02.12.2019 
extending the rule $m X=C(m)$ for all $m \in M$, where $(m X) r=C(m) r=C\left(m r^{p^{e}}\right)=$ $m r^{p^{e}} X=m f^{e}(r) X=m(X r)$ for all $r \in R$ and $m \in M$.

When $\mathrm{R}$ is complete local and $F$-finite, it was shown by R. Y. Sharp and Y. Yoshino in [8] and independently by M. Blickle and G. Böckle in [1] that there is a bijective correspondence between the category of left $R\left[X ; f^{e}\right]$-modules that are Artinian as $R$ modules and the category of right $R\left[X ; f^{e}\right]$-modules that are Noetherian as $R$-modules. Sharp and Yoshino used this correspondence to translate known results about Artinian modules equipped with Frobenius maps into results about Noetherian modules equipped with Cartier maps, yet Blickle and Böckle used this correspondence to show how their results about Cartier modules globalize known results about left $R\left[X ; f^{e}\right]$-modules that are Artinian as R-modules. (When $e=1$, we simply drop it from notations.)

In this paper, we consider the case that $R=\mathbb{k} \llbracket x_{1}, \ldots, x_{n} \rrbracket$ is a power series ring over a perfect field $\mathbb{k}$ of prime characteristic $\mathrm{p}$, and we introduce an explicit correspondence between Noetherian $R$-modules equipped with a Cartier map and Artinian R-modules equipped with a Frobenius map which coincides with the correspondences in [8] and [1], more importantly, extends to a computational level (see Theorem 5.3). To do this, we introduce an explicit isomorphism between two modules which are well-known isomorphic modules but an isomorphism has not been given explicitly before (see Lemma 4.2).

\section{Noetherian modules with Cartier maps}

In this section, we give an explicit formulation for Cartier maps over finitely generated modules. To do this, we start with explaining the $F_{*}^{e} R$-module structure of $\operatorname{Hom}_{R}\left(F_{*}^{e} R^{\alpha}, R^{\alpha}\right)$, where $\alpha$ is a positive integer. Let $R=\mathbb{k} \llbracket x_{1}, \ldots, x_{n} \rrbracket$ be a power series ring over an $F$-finite field $\mathbb{k}$ of prime characteristic $p$.

Remark 2.1. Let $\mathcal{C}$ be a base for $\mathbb{k}$ as a $\mathbb{k}^{p^{e}}$-vector space which includes the identity element of $\mathbb{k}$. Then $F_{*}^{e} R$ are free $R$-modules with the basis set

$$
\mathcal{B}=\left\{F_{*}^{e} \lambda x_{1}^{\alpha_{1}} \ldots x_{n}^{\alpha_{n}} \mid 0 \leq \alpha_{1}, \ldots, \alpha_{n}<p^{e}, \lambda \in \mathcal{C}\right\} .
$$

In particular, if $\mathbb{k}$ is perfect then $F_{*}^{e} R$ are free $R$-modules with the basis set

$$
\mathcal{B}=\left\{F_{*}^{e} x_{1}^{\alpha_{1}} \ldots x_{n}^{\alpha_{n}} \mid 0 \leq \alpha_{1}, \ldots, \alpha_{n}<p^{e}\right\} .
$$

Henceforth, in this section $R=\mathbb{k} \llbracket x_{1}, \ldots, x_{n} \rrbracket$ will denote a power series ring over a perfect field $\mathbb{k}$ of prime characteristic $p$.

Lemma 2.2 ([2, Example 3.0.5]). Let $\pi_{e}: F_{*}^{e} R \rightarrow R$ be the projection map onto the free summand $R F_{*}^{e} x_{1}^{p^{e}-1} \ldots x_{n}^{p^{e}-1}$. Then $\operatorname{Hom}_{R}\left(F_{*}^{e} R, R\right)$ is generated by $\pi_{e}$ as an $F_{*}^{e} R$-module.

Proof. For each basis element $F_{*}^{e} x_{1}^{\alpha_{1}} \ldots x_{n}^{\alpha_{n}} \in \mathcal{B}$, the projection map onto the free summand $R F_{*}^{e} x_{1}^{\alpha_{1}} \ldots x_{n}^{\alpha_{n}}$ is defined by the rule

$$
F_{*}^{e} z \cdot \pi_{e}(-)=\pi_{e}\left(F_{*}^{e} z \cdot-\right),
$$

where $z=x_{1}^{p^{e}-1-\alpha_{1}} \ldots x_{n}^{p^{e}-1-\alpha_{n}}$. Since we can obtain all of the projections in this way, the map

$$
\Phi: F_{*}^{e} R \rightarrow \operatorname{Hom}_{R}\left(F_{*}^{e} R, R\right) \text { defined by } \Phi\left(F_{*}^{e} u\right)=\phi_{u},
$$

where $\phi_{u}: F_{*}^{e} R \rightarrow R$ is the $R$-linear map $\phi_{u}(-)=\pi_{e}\left(F_{*}^{e} u-\right)$, is surjective. On the other hand, if $\Phi\left(F_{*}^{e} u\right)=0$ for some $u \in R$, then we have

$$
\phi_{u}\left(F_{*}^{e} r\right)=\pi_{e}\left(F_{*}^{e} u r\right)=F_{*}^{e} u . \pi_{e}\left(F_{*}^{e} r\right)=0 \text { for all } r \in R .
$$

This means that $F_{*}^{e} u$ must be zero, and so $\Phi$ is injective. Hence, $\Phi$ is an $F_{*}^{e} R$ isomorphism. In other words, $\pi_{e}$ generates $\operatorname{Hom}_{R}\left(F_{*}^{e} R, R\right)$ as an $F_{*}^{e} R$-module.

The following lemma extends Lemma 2.2 to free modules. 
Lemma 2.3. For any $\Psi \in \operatorname{Hom}_{R}\left(F_{*}^{e} R^{\alpha}, R^{\alpha}\right)$, there exists an $\alpha \times \alpha$ matrix $U$ with entries in $R$ such that $\Psi(-)=\Pi_{e}\left(F_{*}^{e} U-\right)$ where

$$
\Pi_{e}\left(\begin{array}{c}
F_{*}^{e} v_{1} \\
\vdots \\
F_{*}^{e} v_{\alpha}
\end{array}\right)=\left(\begin{array}{c}
\pi_{e}\left(F_{*}^{e} v_{1}\right) \\
\vdots \\
\pi_{e}\left(F_{*}^{e} v_{\alpha}\right)
\end{array}\right)
$$

for all $\left(F_{*} v_{1}, \ldots, F_{*}^{e} v_{\alpha}\right)^{t} \in F_{*}^{e} R^{\alpha}$, and $\pi_{e} \in \operatorname{Hom}_{R}\left(F_{*}^{e} R, R\right)$ as in Lemma 2.2.

Proof. If $\alpha=1$, by Lemma 2.2, $\operatorname{Hom}_{R}\left(F_{*}^{e} R, R\right)$ is generated as an $F_{*}^{e} R$-module by the map $\pi_{e}$. If $\alpha>1$, we first need to describe elements of $\operatorname{Hom}_{R}\left(F_{*}^{e} R, R^{\alpha}\right)$. Since $\operatorname{Hom}_{R}\left(F_{*}^{e} R, R^{\alpha}\right) \cong \operatorname{Hom}_{R}\left(F_{*}^{e} R, R\right)^{\alpha}$, any $R$-linear map $\varphi \in \operatorname{Hom}_{R}\left(F_{*}^{e} R, R^{\alpha}\right)$ can be expressed as a direct sum of elements of $\operatorname{Hom}_{R}\left(F_{*}^{e} R, R\right)$. Therefore, we have $\varphi(-)=$ $\left(\phi_{1}(-), \ldots, \phi_{\alpha}(-)\right)^{t}$ for some $\phi_{i} \in \operatorname{Hom}_{R}\left(F_{*}^{e} R, R\right)$ where $1 \leq i \leq \alpha$, and by Lemma 2.2, $\varphi(-)=\left(\pi_{e}\left(F_{*}^{e} u_{1}-\right), \ldots, \pi_{e}\left(F_{*}^{e} u_{\alpha}-\right)\right)^{t}$ for some $u_{1}, \ldots, u_{\alpha} \in R$.

Since $\operatorname{Hom}_{R}\left(F_{*}^{e} R^{\alpha}, R^{\alpha}\right) \cong \operatorname{Hom}_{R}\left(F_{*}^{e} R, R^{\alpha}\right)^{\alpha}$, any $\Psi \in \operatorname{Hom}_{R}\left(F_{*}^{e} R^{\alpha}, R^{\alpha}\right)$ can be expressed as a direct sum of elements of $\operatorname{Hom}_{R}\left(F_{*}^{e} R, R^{\alpha}\right)$. Therefore, for any element $\left(v_{1}, \ldots, v_{\alpha}\right)^{t} \in R^{\alpha}$, we have

$$
\Psi\left(\left(F_{*}^{e} v_{1}, \ldots, F_{*}^{e} v_{\alpha}\right)^{t}\right)=\sum_{1 \leq j \leq \alpha} \varphi_{j}\left(F_{*}^{e} v_{j}\right)
$$

for some $\varphi_{j} \in \operatorname{Hom}_{R}\left(F_{*}^{e} R, R^{\alpha}\right)$. By the previous observation of $\operatorname{Hom}_{R}\left(F_{*}^{e} R, R^{\alpha}\right)$, for each $j$, we also have

$$
\varphi_{j}\left(F_{*}^{e} v_{j}\right)=\left(\pi_{e}\left(F_{*}^{e} u_{1 j} v_{j}\right), \ldots, \pi_{e}\left(F_{*}^{e} u_{\alpha j} v_{j}\right)\right)^{t}
$$

for some elements $u_{1 j}, \ldots, u_{\alpha j} \in R$. Thus,

$$
\Psi\left(\begin{array}{c}
F_{*}^{e} v_{1} \\
\vdots \\
F_{*}^{e} v_{\alpha}
\end{array}\right)=\sum_{1 \leq j \leq \alpha}\left(\begin{array}{c}
\pi_{e}\left(F_{*}^{e} u_{1 j} v_{j}\right) \\
\vdots \\
\pi_{e}\left(F_{*}^{e} u_{\alpha j} v_{j}\right)
\end{array}\right) .
$$

Hence, for any $\Psi \in \operatorname{Hom}_{R}\left(F_{*}^{e} R^{\alpha}, R^{\alpha}\right)$, there exists an $\alpha \times \alpha$ matrix $U$ with entries $u_{i j} \in R$ such that $\Psi(-)=\Pi_{e}\left(F_{*}^{e} U-\right)$ where $\Pi_{e}$ takes the components of elements in $F_{*}^{e} R^{\alpha}$ to their images under the map $\pi_{e}$.

Definition 2.4. Let the notation and situation be as in Lemma 2.3. We call the map $\Pi_{e}$ $\left(\pi_{e}\right.$ when $\left.\alpha=1\right)$ in Lemma 2.3 the trace map on $F_{*}^{e} R^{\alpha}$, or just the trace map when the content is clear.

Proposition 2.5. Let $M$ be a finitely generated Cartier module equipped with a Cartier map $C$ which is isomorphic to $R^{\alpha} / \operatorname{Im} A$ for some positive integer $\alpha$ and matrix $A$ with entries in $R$. Then there exists a Cartier module structure $C^{\prime}$ on $R^{\alpha}$ such that the diagram below is commutative

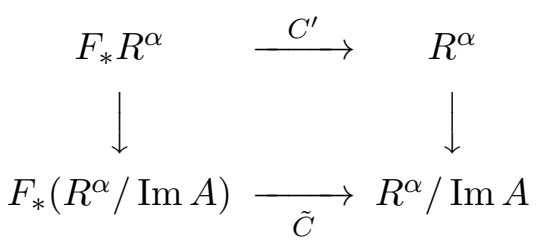

where the vertical arrows are natural surjections and $\tilde{C}$ is isomorphic to $C$.

Proof. Since $F_{*} R$ is a free $R$-module, there exists an $R$-linear map $C^{\prime}: F_{*} R^{\alpha} \rightarrow R^{\alpha}$ such that the Diagram 2.1 is commutative.

Let $M$ be a given finitely generated Cartier module with a Cartier map $C$ which is isomorphic to $R^{\alpha} / \operatorname{Im} A$ for some positive integer $\alpha$ and matrix $A$ with entries in $R$. By Proposition 2.5, there exists a Cartier module structure $C^{\prime}$ on $R^{\alpha}$ such that $C^{\prime}\left(F_{*} \operatorname{Im} A\right) \subseteq$ 
$\operatorname{Im} A$. Then by Lemma 2.3, there exists an $\alpha \times \alpha$ matrix $U$ with entries in $R$ such that $C^{\prime}(-)=\Pi\left(F_{*} U-\right)$ whose restriction on $M$ is $C$.

\section{Artinian modules with Frobenius maps}

In this section, we give an explicit formulation for Frobenius maps over Artinian modules. Let $E=E_{R}(R / \mathfrak{m})$ be the injective hull of the residue field of $R$.

Remark 3.1. Following [3, 13.5.3 Example], $E$ is isomorphic to the module of inverse polynomials $\mathbb{k}\left[x_{1}^{-}, \ldots, x_{s}^{-}\right]$whose $R$-module structure is extended from the following rule

$$
\begin{aligned}
\left(\lambda x_{1}^{\alpha_{1}} \ldots x_{s}^{\alpha_{s}}\right) & \left(\mu x_{1}^{-\nu_{1}} \ldots x_{s}^{-\nu_{s}}\right) \\
& = \begin{cases}\lambda \mu x_{1}^{-\nu_{1}+\alpha_{1}} \ldots x_{s}^{-\nu_{s}+\alpha_{s}} & \text { if } \alpha_{i}<\nu_{i} \text { for all } i \\
0 & \text { if } \alpha_{i} \geq \nu_{i} \text { for any } i\end{cases}
\end{aligned}
$$

for all $\lambda, \mu \in \mathbb{k}$, non-negative integers $\alpha_{1}, \ldots, \alpha_{s}$, and positive integers $\nu_{1}, \ldots, \nu_{s}$. Therefore, $E$ has a natural left $R\left[X ; f^{e}\right]$-module structure by extending additively the action

$$
T\left(\lambda x_{1}^{\alpha_{1}} \ldots x_{s}^{\alpha_{s}}\right)=\lambda^{p^{e}} x_{1}^{-p^{e} \nu_{1}} \ldots, x_{s}^{-p^{e} \nu_{s}},
$$

where $T=X$, for all $\lambda \in \mathbb{k}$ and positive integers $\nu_{1}, \ldots, \nu_{s}$. Notice that $T: E \rightarrow E$ defines and $e$-th Frobenius map. We can also extend this natural left $R\left[X ; f^{e}\right]$-module structure on $E$ to $E^{\alpha}$ which is given by

$$
T\left(\begin{array}{c}
a_{1} \\
\vdots \\
a_{\alpha}
\end{array}\right)=\left(\begin{array}{c}
T a_{1} \\
\vdots \\
T a_{\alpha}
\end{array}\right)
$$

for all $a_{1}, \ldots, a_{\alpha} \in E$, and we call this $T$ as the natural Frobenius on $E^{\alpha}$.

Let $M$ be an $R$-module. The Frobenius functor $F_{R}$ from the category of $R$-modules to itself is defined by $F_{R}(M):=F_{*} R \otimes_{R} M$ where $F_{R}(M)$ acquires its $R$-module structure via the identification of $F_{*} R$ with $R$. The resulting $\left(F_{*} R, R\right)$-bimodule structure on $F_{R}(M)$ satisfies

$$
s \cdot\left(F_{*} r \otimes m\right):=F_{*} s r \otimes m \text { and }\left(F_{*} r \otimes m\right) \cdot s:=F_{*} s^{p} r \otimes m=F_{*} r \otimes s m
$$

for all $r, s \in R$ and $m \in M$. The $e$-th iteration of $F_{R}$ is denoted by $F_{R}^{e}$, and it is clearly given by $F_{R}^{e}(M)=F_{*}^{e} R \otimes_{R} M$. Regularity of $R$ implies that the Frobenius functor is exact.

Remark 3.2. Following [5, Section 3], let $\mathfrak{C}^{e}$ be the category of Artinian left $R\left[X ; f^{e}\right]$ modules and $\mathfrak{D}^{e}$ be the category of $R$-linear maps $M \rightarrow F_{R}^{e}(M)$ where $M$ is a Noetherian $R$-module and a morphism between $M \rightarrow F_{R}^{e}(M)$ and $N \rightarrow F_{R}^{e}(N)$ is a commutative diagram of $R$-linear maps

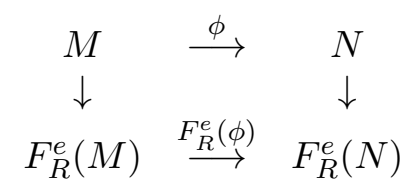

We define the functor $\Delta^{e}: \mathfrak{C}^{e} \rightarrow \mathfrak{D}^{e}$ as follows: given an $e$-th Frobenius map $\theta: M \rightarrow M$, we can obtain an $R$-linear map $\phi: F_{*}^{e} R \otimes M \rightarrow M$ such that $\phi\left(F_{*} r \otimes m\right)=r \theta(m)$ for all $r \in R, m \in M$. Applying the Matlis duality functor to this map gives the $R$-linear map $M^{\vee} \rightarrow\left(F_{*}^{e} R \otimes M\right)^{\vee} \cong F_{*}^{e} R \otimes M^{\vee}$ where the last isomorphism is described in [7, Lemma 4.1]. Conversely, we define the functor $\Psi^{e}: \mathfrak{D}^{e} \rightarrow \mathfrak{C}^{e}$ as follows: given a Noetherian $R$ module $N$ with an $R$-linear map $N \rightarrow F_{R}^{e}(N)$. Applying the Matlis duality functor to this map gives the $R$-linear map $\varphi: F_{R}^{e}\left(N^{\vee}\right) \cong F_{R}^{e}(N)^{\vee} \rightarrow N^{\vee}$ where the first isomorphism is the composition $F_{R}^{e}\left(N^{\vee}\right) \cong F_{R}^{e}\left(N^{\vee}\right)^{\vee \vee} \cong F_{R}^{e}\left(N^{\vee}\right) \vee \cong F_{R}^{e}(N)^{\vee}$. Then we define the action of $\theta$ on $N^{\vee}$ by defining $\theta(n)=\varphi(1 \otimes n)$ for all $n \in N^{\vee}$. The mutually inverse exact 
functors $\Delta^{e}$ and $\Psi^{e}$ are extensions of the Matlis duality functor which also keeps track of Frobenius actions.

Given an Artininan $R$-module $M$, we can embed $M$ in $E^{\alpha}$ for some positive integer $\alpha$, we can then embed $\operatorname{Coker}\left(M \hookrightarrow E^{\alpha}\right)$ in $E^{\beta}$ for some positive integer $\beta$. Continuing in this way, we get an injective resolution

$$
0 \rightarrow M \rightarrow E^{\alpha} \stackrel{A^{t}}{\rightarrow} E^{\beta} \rightarrow \cdots
$$

of $M$, where $A$ is an $\alpha \times \beta$ matrix with entries in $R$ since $\operatorname{Hom}_{R}\left(E^{\alpha}, E^{\beta}\right) \cong \operatorname{Hom}_{R}\left(S^{\alpha}, R^{\beta}\right)$, and so $M \cong \operatorname{Ker} A^{t}$.

Proposition 3.3 ([6, Proposition 2.1]). Let $M \cong \operatorname{Ker} A^{t}$ be an Artininan R-module where $A$ is an $\alpha \times \beta$ matrix with entries in $R$. For a given e-th Frobenius map on $M, \Delta^{e}(M) \in$ $\operatorname{Hom}_{R}\left(\right.$ Coker $A$, Coker $\left.A^{\left[p^{e}\right]}\right)$ and is given by an $\alpha \times \alpha$ matrix $U$ such that $U \operatorname{Im} A \subseteq \operatorname{Im} A^{\left[p^{e}\right]}$, conversely any such $U$ defines an $R\left[X ; f^{e}\right]$-module structure on $M$ which is given by the restriction to $M$ of the Frobenius map $X: E^{\alpha} \rightarrow E^{\alpha}$ defined by $X(a)=U^{t} T(a)$ for all $a \in E^{\alpha}$.

\section{An explicit isomorphism}

Henceforth $R=\mathbb{k} \llbracket x_{1}, \ldots, x_{n} \rrbracket$ will denote a power series ring over a perfect field $\mathbb{k}$ of prime characteristic $p$, and we will identify $E$ with the module of inverse polynomials. Since $F_{*} E$ is the injective hull of the residue field of $F_{*} R$, by [4, Theorem A25], we know that $\operatorname{Hom}_{R}\left(F_{*} R, E\right) \cong F_{*} E$ as $F_{*} R$-modules. In this section, we define an explicit isomorphism between $\operatorname{Hom}_{R}\left(F_{*} R, E\right)$ and $F_{*} E$.

Notation 4.1. Let $k, k_{1}, k_{2}$ be integers. We will write

(1) $\bar{x}$ to denote $x_{1} \ldots x_{n}$,

(2) $\bar{\alpha}, \bar{\alpha}+\bar{\beta}$ and $k \bar{\alpha}$ to denote the $n$-tuples $\left(\alpha_{1}, \ldots, \alpha_{n}\right),\left(\alpha_{1}+\beta_{1}, \ldots, \alpha_{n}+\beta_{n}\right)$ and $\left(k \alpha_{1}, \ldots, k \alpha_{n}\right)$, respectively,

(3) $\bar{x}^{\bar{\alpha}}$ to denote $x_{1}^{\alpha_{1}} \ldots x_{n}^{\alpha_{n}}$, and $\bar{x}^{k}$ to denote $x_{1}^{k} \ldots x_{n}^{k}$,

(4) $k_{1}<\bar{\alpha}<k_{2}$ to mean that $k_{1}<\alpha_{i}<k_{2}$ for each $i$,

(5) $r_{\bar{\alpha}}$ and $r_{\bar{k}}$ to denote the elements of $R$ indexed with the $n$-tuples $\bar{\alpha}$ and $(k, \ldots, k)$, respectively,

(6) $-\nu$ to denote $n$-tuples $\left(\nu_{1}, \ldots, \nu_{n}\right)$.

By Remark 2.1, $F_{*}^{e} R$ is a free $R$-module with the basis set $\mathcal{B}=\left\{F_{*}^{e} x_{1}^{\alpha_{1}} \ldots x_{n}^{\alpha_{n}} \mid 0 \leq\right.$ $\left.\alpha_{1}, \ldots, \alpha_{n}<p^{e}\right\}$. Therefore, an $R$-linear map from $F_{*} R$ to any other $R$-module is simply a choice of where to send these basis elements.

Lemma 4.2. The map $\Phi: \operatorname{Hom}_{R}\left(F_{*} R, E\right) \rightarrow F_{*} E$ given by

$$
\Phi(g)=\sum_{0 \leq \bar{\alpha}<p} F_{*}\left[\bar{x}^{p-1-\bar{\alpha}} T\left(g\left(F_{*} \bar{x}^{\bar{\alpha}}\right)\right)\right]
$$

for all $g \in \operatorname{Hom}_{R}\left(F_{*} R, E\right)$, where $T$ is the natural Frobenius map on $E$, is an $F_{*} R$ isomorphism.

Proof. By the definitions of $T$ and $g$, it can easily be seen that $\Phi$ is well-defined and additive. For any $r \in R$, we further have the following

$$
\begin{aligned}
r \Phi(g) & =\sum_{0 \leq \bar{\alpha}<p} F_{*}\left[\bar{x}^{p-1-\bar{\alpha}} r^{p} T\left(g\left(F_{*} \bar{x}^{\bar{\alpha}}\right)\right)\right] \\
& =\sum_{0 \leq \bar{\alpha}<p} F_{*}\left[\bar{x}^{p-1-\bar{\alpha}} T\left(r g\left(F_{*} \bar{x}^{\bar{\alpha}}\right)\right)\right]=\Phi(r g)
\end{aligned}
$$


which means that $\Phi$ is $R$-linear. Thus, for $F_{*} R$-linearity of $\Phi$, since $F_{*} R$ is a free $R$-module, it is enough to show that $F_{*} \bar{x}^{\bar{\beta}} \Phi(g)=\Phi\left(F_{*} \bar{x}^{\bar{\beta}} g\right)$ for any basis element $F_{*} \bar{x}^{\bar{\beta}} \in \mathcal{B}$, and so we will show that the right hand sides of following equations are equal.

$$
\begin{aligned}
& F_{*} \bar{x}^{\bar{\beta}} \Phi(g)=\sum_{0 \leq \bar{\alpha}<p} F_{*}\left[\bar{x}^{p-1-\bar{\alpha}+\bar{\beta}} T\left(g\left(F_{*} \bar{x}^{\bar{\alpha}}\right)\right)\right], \\
& \Phi\left(F_{*} \bar{x}^{\bar{\beta}} g\right)=\sum_{0 \leq \bar{\alpha}<p} F_{*}\left[\bar{x}^{p-1-\bar{\alpha}} T\left(g\left(F_{*} \bar{x}^{\bar{\alpha}+\bar{\beta}}\right)\right)\right] .
\end{aligned}
$$

Moreover, since $F_{*} \bar{x}^{\bar{\beta}}=F_{*} x_{1}^{\beta_{1}} \cdots F_{*} x_{n}^{\beta_{n}}$, it is enough to show that

$$
\begin{aligned}
F_{*} x_{i}^{\beta_{i}} \Phi(g) & =\sum_{0 \leq \bar{\alpha}<p} F_{*}\left[x_{1}^{p-1-\alpha_{1}} \ldots x_{i}^{p-1-\alpha_{i}+\beta_{i}} \ldots x_{n}^{p-1-\alpha_{n}} T\left(g\left(F_{*} \bar{x}^{\bar{\alpha}}\right)\right)\right] \\
& =\sum_{0 \leq \bar{\alpha}<p} F_{*}\left[\bar{x}^{p-1-\bar{\alpha}} T\left(g\left(F_{*} x_{1}^{\alpha_{1}} \ldots x_{i}^{\alpha_{i}+\beta_{i}} \ldots x_{n}^{\alpha_{n}}\right)\right)\right]=\Phi\left(F_{*} x_{i}^{\beta_{i}} g\right)
\end{aligned}
$$

for each $F_{*} x_{i}^{\beta_{i}}$. To do that we will show the following sets are the same

$$
\begin{gathered}
S_{1}=\left\{F_{*}\left[\bar{x}^{p-1-\bar{\alpha}} T\left(g\left(F_{*} x_{1}^{\alpha_{1}} \ldots x_{i}^{\alpha_{i}+\beta_{i}} \ldots x_{n}^{\alpha_{n}}\right)\right)\right] \mid 0 \leq \alpha_{i}<p\right\}, \\
S_{2}=\left\{F_{*}\left[x_{1}^{p-1-\alpha_{1}} \ldots x_{i}^{p-1-\alpha_{i}+\beta_{i}} \ldots x_{n}^{p-1-\alpha_{n}} T\left(g\left(F_{*} \bar{x}^{\bar{\alpha}}\right)\right)\right] \mid 0 \leq \alpha_{i}<p\right\} .
\end{gathered}
$$

In the case that $\alpha_{i}+\beta_{i}<p$,

$$
\begin{gathered}
\left\{F_{*}\left[\bar{x}^{p-1-\bar{\alpha}} T\left(g\left(F_{*} x_{1}^{\alpha_{1}} \ldots x_{i}^{\alpha_{i}+\beta_{i}} \ldots x_{n}^{\alpha_{n}}\right)\right)\right] \mid 0 \leq \alpha_{i}<p-\beta_{i}\right\}= \\
\left\{F_{*}\left[x_{1}^{p-1-\alpha_{1}} \ldots x_{i}^{p-1-\alpha_{i}+\beta_{i}} \ldots x_{n}^{p-1-\alpha_{n}} T\left(g\left(F_{*} \bar{x}^{\bar{\alpha}}\right)\right)\right] \mid \beta_{i} \leq \alpha_{i}<p\right\}
\end{gathered}
$$

since substituting $\alpha_{i}$ with $\alpha_{i}+\beta_{i}$ in the latter set gives us the former set. On the other hand, in the case that $\alpha_{i}+\beta_{i} \geq p$,

$$
\begin{gathered}
\left\{F_{*}\left[\bar{x}^{p-1-\bar{\alpha}} T\left(g\left(F_{*} x_{1}^{\alpha_{1}} \ldots x_{i}^{\alpha_{i}+\beta_{i}} \ldots x_{n}^{\alpha_{n}}\right)\right)\right] \mid p-\beta_{i} \leq \alpha_{i}<p\right\}= \\
\left\{F_{*}\left[x_{1}^{p-1-\alpha_{1}} \ldots x_{i}^{p-1-\alpha_{i}+\beta_{i}} \ldots x_{n}^{p-1-\alpha_{n}} T\left(g\left(F_{*} \bar{x}^{\bar{\alpha}}\right)\right)\right] \mid 0 \leq \alpha_{i}<\beta_{i}\right\}
\end{gathered}
$$

since for each $k \in\left\{0, \ldots, \beta_{i}-1\right\}$, where $\alpha_{i}+\beta_{i}=p+k$ (i.e. $p-\alpha_{i}=\beta_{i}-k$ ),

$$
\begin{gathered}
F_{*}\left[\bar{x}^{p-1-\bar{\alpha}} T\left(g\left(F_{*} x_{1}^{\alpha_{1}} \ldots x_{i}^{\alpha_{i}+\beta_{i}} \ldots x_{n}^{\alpha_{n}}\right)\right)\right]= \\
F_{*}\left[x_{1}^{p-1-\alpha_{1}} \ldots x_{i}^{p-1-\alpha_{i}+p} \ldots x_{n}^{p-1-\alpha_{n}} T\left(g\left(F_{*} x_{1}^{\alpha_{1}} \ldots x_{i}^{k} \ldots x_{n}^{\alpha_{n}}\right)\right)\right]= \\
F_{*}\left[x_{1}^{p-1-\alpha_{1}} \ldots x_{i}^{p-1-k+\beta_{i}} \ldots x_{n}^{p-1-\alpha_{n}} T\left(g\left(F_{*} x_{1}^{\alpha_{1}} \ldots x_{i}^{k} \ldots x_{n}^{\alpha_{n}}\right)\right)\right] .
\end{gathered}
$$

Therefore, $S_{1}=S_{2}$, and so the right hand sides of (4.2) and (4.3) are equal.

For injectivity of $\Phi$, we first need the following. For any $g \in \operatorname{Hom}_{R}\left(F_{*} R, E\right)$, we have $g\left(F_{*} \bar{x}^{\bar{\alpha}}\right) \in \mathbb{k}\left[x_{1}^{-}, \ldots, x_{n}^{-}\right]$, and so $g\left(F_{*} \bar{x}^{\bar{\alpha}}\right)$ is a finite $\mathbb{k}$-linear combination of monomials $x_{1}^{-\nu_{1}} \ldots x_{n}^{-\nu_{n}}$, where $\nu_{i}$ 's are positive integers. Therefore, for each $F_{*} \bar{x}^{\bar{\alpha}} \in \mathcal{B}$, $F_{*}\left[\bar{x}^{p-1-\bar{\alpha}} T\left(g\left(F_{*} \bar{x}^{\bar{\alpha}}\right)\right)\right]$ is a finite $\mathbb{k}$-linear combination of monomials

$$
F_{*} x_{1}^{p-1-\alpha_{1}-p \nu_{1}} \ldots x_{n}^{p-1-\alpha_{n}-p \nu_{n}} .
$$

This means that

$$
\Phi(g)=\sum_{0 \leq \bar{\alpha}<p} F_{*}\left[\bar{x}^{p-1-\bar{\alpha}} T\left(g\left(F_{*} \bar{x}^{\bar{\alpha}}\right)\right)\right]=\sum_{0 \leq \bar{\alpha}<p}\left(\sum_{0<\bar{\nu}<\infty} \lambda_{\nu} F_{*} \bar{x}^{p-1-\bar{\alpha}-p \bar{\nu}}\right) .
$$

Therefore, $\Phi(g)=0$ if and only if $\lambda_{\nu}=0$ for all $\bar{\nu}>0$ since $p-1-\bar{\alpha}<p$ implies that $p-1-\bar{\alpha}-p \bar{\nu}<0$ and the terms $F_{*} \bar{x}^{p-1-\bar{\alpha}-p \bar{\nu}} \neq F_{*} \bar{x}^{p-1-\bar{\beta}-p \bar{\mu}}$ unless $\bar{\alpha}=\bar{\beta}$ and $\bar{\mu}=\bar{\nu}$ at the same time. Hence,

$$
\begin{aligned}
\Phi(g)=0 & \Leftrightarrow T\left(g\left(F_{*} \bar{x}^{\bar{\alpha}}\right)\right)=0 \text { for all } 0 \leq \bar{\alpha}<p \\
& \Leftrightarrow g\left(F_{*} \bar{x}^{\bar{\alpha}}\right)=0 \text { for all } 0 \leq \bar{\alpha}<p \\
& \Leftrightarrow g=0 .
\end{aligned}
$$


For surjectivity of $\Phi$, we take an element $F_{*} e$ of $F_{*} \mathbb{k}\left[x_{1}^{-}, \ldots, x_{n}^{-}\right]$. We know that it can be written as a finite sum of terms $F_{*} \mu x_{1}^{-\nu_{1}} \ldots x_{n}^{-\nu_{n}}$, where $\mu \in \mathbb{k}$, and these terms can be written as

$$
\begin{aligned}
F_{*} \mu x_{1}^{-\nu_{1}} \ldots x_{n}^{-\nu_{n}} & =F_{*} x_{1}^{k_{1}} \ldots x_{n}^{k_{n}} F_{*} \lambda^{p} x_{1}^{-p \beta_{1}} \ldots x_{n}^{-p \beta_{n}} \\
& =F_{*} x_{1}^{k_{1}} \ldots x_{n}^{k_{n}} F_{*} T\left(\lambda x_{1}^{-\beta_{1}} \ldots x_{n}^{-\beta_{n}}\right),
\end{aligned}
$$

where $\mu=\lambda^{p}$, and for each $i, k_{i}=p \beta_{i}-\nu_{i}$ and $0 \leq k_{i}<p$. Now we rewrite $F_{*} e$ as a finite sum

$$
\sum_{0 \leq k_{1}, \ldots, k_{n}<p} F_{*} x_{1}^{k_{1}} \ldots x_{n}^{k_{n}} F_{*} T\left(e_{k_{1}, \ldots, k_{n}}\right)
$$

where $e_{k_{1}, \ldots, k_{n}} \in \mathbb{k}\left[x_{1}^{-}, \ldots, x_{n}^{-}\right]$, and we choose a map $g \in \operatorname{Hom}_{R}\left(F_{*} R, E\right)$ which sends $F_{*} x_{1}^{p-1-k_{1}} \ldots x_{n}^{p-1-k_{n}}$ to $e_{k_{1}, \ldots, k_{n}}$. This means that $\Phi(g)=F_{*} e$, i.e. $\Phi$ is surjective. Hence, it is an isomorphism of $F_{*} R$-modules.

\section{The correspondence}

In this section, we introduce our explicit correspondence between finitely generated Cartier modules and Artinian modules equipped with a Frobenius map via the Matlis duality. We use Lemma 4.2 repeatedly to give our explicit proofs.

Proposition 5.1. Let $\alpha$ be a non-negative integer. There is a bijective correspondence between $\operatorname{Hom}_{R}\left(F_{*} R^{\alpha}, R^{\alpha}\right)$ and $\operatorname{Hom}_{R}\left(E^{\alpha}, F_{*} E^{\alpha}\right)$ such that the trace map $\Pi$ on $F_{*} R^{\alpha}$ corresponds to the natural Frobenius map $T$ on $E^{\alpha}$ and $\Pi\left(F_{*} U-\right)$ corresponds to $U^{t} T$ for any $\alpha \times \alpha$ matrix $U$ with entries in $R$.

Proof. We start by identifying $\operatorname{Hom}_{R}\left(F_{*} R, E\right)$ with $F_{*} E$ using the isomorphism $\Phi$ defined in Lemma 4.2. Then we first assume that $\alpha=1$ and let $\phi: F_{*} R \rightarrow R$ be a Cartier map. We know that there is an element $u \in R$ such that $\phi(-)=\pi\left(F_{*} u-\right)$. Applying the Matlis duality functor to this map gives us $\operatorname{Hom}_{R}(R, E) \stackrel{f \mapsto f \circ \phi}{\longrightarrow} \operatorname{Hom}_{R}\left(F_{*} R, E\right)$. Next we use the isomorphism $E \stackrel{e \mapsto f_{e}}{\longrightarrow} \operatorname{Hom}_{R}(R, E)$, where $f_{e}(1)=e$, to get the following composition:

$$
\begin{aligned}
& E \rightarrow \operatorname{Hom}_{R}(R, E) \rightarrow \operatorname{Hom}_{R}\left(F_{*} R, E\right) \rightarrow \quad F_{*} E \\
& e \mapsto \quad f_{e} \mapsto \quad f_{e} \circ \phi \mapsto \quad \Phi\left(f_{e} \circ \phi\right) \\
& \begin{aligned}
\Phi\left(f_{e} \circ \phi\right) & =\Phi\left(f_{e} \circ \pi\left(F_{*} u-\right)\right)\left(\text { by } F_{*} R \text {-linearity of } \Phi\right) \\
& =F_{*} u \Phi\left(f_{e} \circ \pi\right)=F_{*} u \sum_{0 \leq \bar{\alpha}<p} F_{*}\left[\bar{x}^{p-1-\bar{\alpha}} T\left(f_{e} \circ \pi\left(F_{*} \bar{x}^{\bar{\alpha}}\right)\right)\right] \\
& =F_{*} u\left[F_{*} T\left(f_{e} \circ \pi\left(F_{*} \bar{x}^{p-1}\right)\right)\right]=F_{*} u\left[F_{*} T\left(f_{e}(1)\right)\right] \\
& =F_{*} u F_{*} T(e)=F_{*}(u T(e)) .
\end{aligned}
\end{aligned}
$$

Therefore, the composition above gives us the Frobenius map $u T$ on $E$. In particular, if $u=1$ we get the natural Frobenius $T$ on $E$.

We now assume that $\alpha>1$ and let $\phi: F_{*} R^{\alpha} \rightarrow R^{\alpha}$ be a Cartier map. We know that there is an $\alpha \times \alpha$ matrix $U$ with entries $\left(u_{i j}\right)_{1 \leq i, j \leq \alpha}$ in $R$ such that $\phi(-)=\Pi\left(F_{*} U-\right)$. Applying the Matlis duality functor to this map gives $\operatorname{Hom}_{R}\left(R^{\alpha}, E\right) \stackrel{f \mapsto f \circ \phi}{\longrightarrow} \operatorname{Hom}_{R}\left(F_{*} R^{\alpha}, E\right)$. 
Then we get the following composition

$$
\begin{array}{ccc}
E^{\alpha} & F_{*} E^{\alpha} \\
a=\left(a_{1}, \ldots, a_{\alpha}\right)^{t} & -\rightarrow & \left(\Phi\left(f_{a} \circ \phi \circ \epsilon_{1}\right), \ldots, \Phi\left(f_{a} \circ \phi \circ \epsilon_{\alpha}\right)\right)^{t} \\
I & \operatorname{Hom}_{R}\left(R^{\alpha}, E\right) \\
\operatorname{Hom}_{R}(R, E)^{\alpha} & \left(f_{a} \circ \phi \circ \epsilon_{1}, \ldots, f_{a} \circ \phi \circ \epsilon_{\alpha}\right)^{t} \\
\left(f_{a_{1}}, \ldots, f_{a_{\alpha}}\right)^{t} & & \stackrel{\uparrow}{I} \\
\operatorname{Hom}_{R}\left(F_{*} R^{\alpha}, E\right) & \longmapsto & \operatorname{Hom}_{R}\left(F_{*} R, E\right)^{\alpha} \\
f_{a} & & f_{a} \circ \phi
\end{array}
$$

where $a \in E^{\alpha}$ and we use the following obvious isomorphisms

$$
E^{\alpha} \rightarrow \operatorname{Hom}_{R}(R, E)^{\alpha} \text { given by }\left(a_{1}, \ldots, a_{\alpha}\right)^{t} \mapsto\left(f_{a_{1}}, \ldots, f_{a_{\alpha}}\right)^{t}
$$

such that $f_{a_{i}}(1)=a_{i}$ for each $i$,

$$
\operatorname{Hom}_{R}(R, E)^{\alpha} \rightarrow \operatorname{Hom}_{R}\left(R^{\alpha}, E\right) \text { given by }\left(g_{1}, \ldots, g_{\alpha}\right)^{t} \mapsto g
$$

such that $g\left(e_{i}\right)=g_{i}(1)$ for each elementary vector $e_{i}$, and

$$
\operatorname{Hom}_{R}\left(F_{*} R^{\alpha}, E\right) \rightarrow \operatorname{Hom}_{R}\left(F_{*} R, E\right)^{\alpha} \text { given by } h \mapsto\left(h \circ \epsilon_{1}, \ldots, h \circ \epsilon_{\alpha}\right)^{t}
$$

such that the map $\epsilon_{i}: F_{*} R \rightarrow F_{*} R^{\alpha}$ given by $F_{*} r \mapsto F_{*} r F_{*} e_{i}$ is the canonical injection for each $i$. Then for a fixed $i$ where $1 \leq i \leq \alpha$, we have

$$
\Phi\left(f_{a} \circ \phi \circ \epsilon_{i}\right)=\sum_{0 \leq \bar{\alpha}<p} F_{*}\left[\bar{x}^{p-1-\bar{\alpha}} T\left(f_{a}\left(\Pi\left(F_{*} U \epsilon_{i}\left(F_{*} \bar{x}^{\bar{\alpha}}\right)\right)\right)\right)\right]
$$

Since

$$
\begin{aligned}
f_{a}\left(\Pi\left(F_{*} U \epsilon_{i}\left(F_{*} \bar{x}^{\bar{\alpha}}\right)\right)\right) & =f_{a}\left(\Pi\left(\left(F_{*} u_{1 i} \bar{x}^{\bar{\alpha}}, \ldots, F_{*} u_{\alpha i} \bar{x}^{\bar{\alpha}}\right)^{t}\right)\right) \\
& =f_{a}\left(\left(\pi\left(F_{*} u_{1 i} \bar{x}^{\bar{\alpha}}\right), \ldots, \pi\left(F_{*} u_{\alpha i} \bar{x}^{\bar{\alpha}}\right)\right)^{t}\right) \\
& =\sum_{1 \leq j \leq \alpha} f_{a_{j}}\left(\pi\left(F_{*} u_{j i} \bar{x}^{\bar{\alpha}}\right)\right),
\end{aligned}
$$

we have

$$
\Phi\left(f_{a} \circ \phi \circ \epsilon_{i}\right)=\sum_{1 \leq j \leq \alpha}\left(\sum_{0 \leq \bar{\alpha}<p} F_{*}\left[\bar{x}^{p-1-\bar{\alpha}} T\left(f_{a_{j}}\left(\pi\left(F_{*} u_{j i} \bar{x}^{\bar{\alpha}}\right)\right)\right)\right]\right) .
$$

Then by definition of $\Phi$,

$$
\begin{aligned}
\Phi\left(f_{a} \circ \phi \circ \epsilon_{i}\right) & =\sum_{1 \leq j \leq \alpha} F_{*} u_{j i} \Phi\left(f_{a_{j}} \circ \pi\right) \\
& =\sum_{1 \leq j \leq \alpha} F_{*} u_{j i} F_{*} T\left(f_{a_{j}}\left(\pi\left(\bar{x}^{p-1}\right)\right)\right) \\
& =\sum_{1 \leq j \leq \alpha} F_{*} u_{j i} T\left(a_{j}\right)=F_{*}\left[\sum_{1 \leq j \leq \alpha} u_{j i} T\left(a_{j}\right)\right] .
\end{aligned}
$$

Therefore, for $1 \leq i \leq \alpha$

$$
\left(\begin{array}{c}
\Phi\left(f_{a} \circ \phi \circ \epsilon_{1}\right) \\
\vdots \\
\Phi\left(f_{a} \circ \phi \circ \epsilon_{\alpha}\right)
\end{array}\right)=\left(\begin{array}{c}
F_{*}\left[\sum_{1 \leq j \leq \alpha} u_{j 1} T\left(a_{j}\right)\right] \\
\vdots \\
F_{*}\left[\sum_{1 \leq j \leq \alpha} u_{j \alpha} T\left(a_{j}\right)\right]
\end{array}\right)
$$

which is equal to

$$
F_{*}\left[U^{t}\left(T\left(\left(a_{1}, \ldots, a_{\alpha}\right)^{t}\right)\right]=F_{*}\left[U^{t} T(a)\right] .\right.
$$

Hence, the composition above gives us the Frobenius map $U^{t} T$ on $E^{\alpha}$. In particular, if $U$ is the identity matrix we get the natural Froebenius $T$ on $E^{\alpha}$. 
The construction above gives us a map $\Omega: \operatorname{Hom}_{R}\left(F_{*} R^{\alpha}, R^{\alpha}\right) \rightarrow \operatorname{Hom}_{R}\left(E^{\alpha}, F_{*} E^{\alpha}\right)$ defined by $\Omega(\phi)=\Theta$ such that $\phi(-)=\Pi\left(F_{*} U-\right)$ and $\Theta(-)=F_{*} U^{t} T(-)$. We claim that this map actually is an $F_{*} R$-linear isomorphism. Let $\Omega\left(F_{*} r . \phi\right)=\Theta^{\prime}$ for any $r \in$ $R$. Then since $\left(F_{*} r . \phi\right)(-)=\phi\left(F_{*} r-\right)=\Pi\left(F_{*} U r-\right)$, we have $\Theta^{\prime}(-)=F_{*}(U r)^{t} T(-)=$ $F_{*} r F_{*} U^{t} T(-)=F_{*} r \Theta(-)$, i.e. $\Omega\left(F_{*} r . \phi\right)=\Theta^{\prime}=F_{*} r \Theta=F_{*} r \Omega(\phi)$, and so $\Omega$ is $F_{*} r$-linear. Surjectivity of $\Omega$ is clear since for any Frobenius map $\Theta(-)=F_{*} U^{t} T(-)$ we can define a Cartier map $\phi(-)=\Pi\left(F_{*} U-\right)$. We also have $\Omega(\phi)=0 \Rightarrow U^{t} T=0 \Rightarrow U=0 \Rightarrow \phi=0$, because if any entry of $U$ was non zero there would be non zero elements in the image of $U^{t} T$, i.e. $\Omega$ is injective. This means that we get the promised bijective correspondence.

Next we see that the Matlis duality functor $(-)^{\vee}=\operatorname{Hom}_{R}(-, E)$ commutes with $F_{*}(-)$ (cf. Lemma 5.1 in [1]).

Lemma 5.2. Let $M$ be a finitely generated or an Artinian $R$-module. Then $F_{*} M^{\vee} \cong$ $\left(F_{*} M\right)^{\vee}$.

Proof. We first assume that $M$ is finitely generated. Then $M$ has a presentation $\cdots \rightarrow$ $R^{\beta} \stackrel{A}{\rightarrow} R^{\alpha} \rightarrow M \rightarrow 0$ where $A$ is an $\alpha \times \beta$ matrix with entries in $R$. If we apply the Matlis dual to this presentation we get $0 \rightarrow M^{\vee} \hookrightarrow E^{\alpha} \stackrel{A^{t}}{\rightarrow} E^{\beta} \rightarrow \ldots$ So $M^{\vee}=\operatorname{Ker} A^{t}=$ $\operatorname{Ann}_{E^{\alpha}} A^{t}$. On the other hand, $F_{*} M$ has a presentation $\cdots \rightarrow F_{*} R^{\beta} \stackrel{F_{*} A}{\longrightarrow} F_{*} R^{\alpha} \rightarrow F_{*} M \rightarrow$ 0 . Then if we apply the Matlis dual again and identify $\operatorname{Hom}_{R}\left(F_{*} R, E\right)$ with $F_{*} E$ using the isomorphism $\Phi$ defined in Lemma 4.2 , we get $0 \rightarrow\left(F_{*} M\right)^{\vee} \hookrightarrow F_{*} E^{\alpha} \stackrel{F_{*} A^{t}}{\longrightarrow} F_{*} E^{\beta} \rightarrow \ldots$, and so $\left(F_{*} M\right)^{\vee}=\operatorname{Ker} F_{*} A^{t}=\operatorname{Ann}_{F_{*} E^{\alpha}} F_{*} A^{t}=F_{*}\left(\operatorname{Ann}_{E^{\alpha}} A^{t}\right)=F_{*} M^{\vee}$.

If now $M$ is Artinian, we know that $M^{\vee}$ is Noetherian and $M \cong M^{\vee \vee}$. Then it follows from the first assumption, $F_{*} M^{\vee} \cong\left(F_{*} M^{\vee}\right)^{\vee \vee} \cong\left(F_{*} M^{\vee \vee}\right)^{\vee} \cong\left(F_{*} M\right)^{\vee}$.

Next theorem extends Proposition 5.2 in [1] to a computational level.

Theorem 5.3. The Matlis duality functor induces a bijective correspondence between finitely generated Cartier modules and Artinian modules equipped with Frobenius maps given as follows: if $M$ is a finitely generated Cartier module with a square matrix $U$ defining the Cartier module structure on $M$, then the corresponding Artinian module is $M^{\vee}$ with the corresponding Frobenius map $U^{t} T$.

Proof. Let $(M, C)$ be a finitely generated Cartier module with a square matrix $U$ defining Cartier module structure on $M$. Then we have a presentation of $M$ as follows $\cdots \rightarrow R^{\beta} \stackrel{A}{\rightarrow}$ $R^{\alpha} \rightarrow M \rightarrow 0$ and the following commutative diagram with exact rows

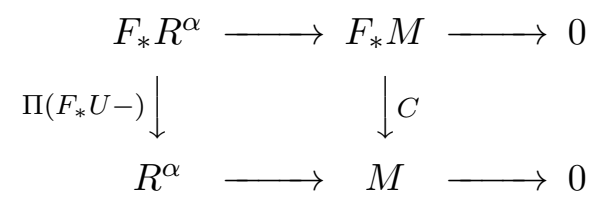

where $C$ is induced by $\Pi\left(F_{*} U-\right)$ on $M$. If we apply the Matlis dual to the diagram above and if we use Lemma 4.2, Proposition 5.1, and Lemma 5.2 we get

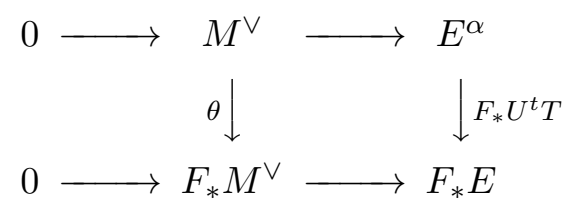

where $\theta$ is the restriction of $F_{*} U^{t} T$ on $M^{\vee}$. The same construction follows the converse.

Question: The theory behind explicit results in this paper works for the case that $R=\mathbb{k} \llbracket x_{1}, \ldots, x_{n} \rrbracket$ is a power series ring over an $F$-finite field $\mathbb{k}$ of prime characteristic $p$. Is it possible to write an explicit isomorphism between $\operatorname{Hom}_{R}\left(F_{*} R, E\right)$ and $F_{*} E$ for the $F$-finite case as in Lemma 4.2 ? 
Acknowledgment. The author thanks Mordechai Katzman for his helpful discussions and valuable suggestions.

\section{References}

[1] M. Blickle and G. Böckle, Cartier modules: finiteness results, J. Reine Angew. Math. 661, 85-123, 2011.

[2] M. Blickle and K. Schwede, $p^{-1}$-linear maps in algebra and geometry, in: Comm. Algebra, 123-205, Springer, New York, 2013.

[3] M.P. Brodmann and R.Y. Sharp, Local Cohomology: An Algebraic Introduction with Geometric Applications, Second Ed., Cambridge University Press, 2013.

[4] S. Iyengar, G.J. Leuschke, A. Leykin, C. Miller, E. Miller, A.K. Singh, and U. Walther, Twenty-Four Hours of Local Cohomology, American Mathematical Society, 2007.

[5] M. Katzman, Parameter test ideals of Cohen Macaulay rings, Compos. Math. 144 (4), 933-948, 2008.

[6] M. Katzman and W. Zhang, Annihilators of Artinian modules compatible with a Frobenius map, J. Symbolic Comput. 60, 29-46, 2014.

[7] G. Lyubeznik, F-modules: applications to local cohomology and D-modules in characteristic $p>0$, J. Reine Angew. Math. 491, 65-130, 1997.

[8] R.Y. Sharp and Y. Yoshino, Right and left modules over the Frobenius skew polynomial ring in the F-finite case, Math. Proc. Cambridge Philos. Soc. 150 (3), 419-438, 2011. 